\title{
Variational Tricomplex, Global Symmetries and Conservation Laws of Gauge Systems
}

\author{
Alexey A. SHARAPOV \\ Physics Faculty, Tomsk State University, Lenin ave. 36, Tomsk 634050, Russia \\ E-mail: sharapov@phys.tsu.ru
}

Received July 12, 2016, in final form September 30, 2016; Published online October 03, 2016

http://dx.doi.org/10.3842/SIGMA.2016.098

\begin{abstract}
Using the concept of variational tricomplex endowed with a presymplectic structure, we formulate the general notion of symmetry. We show that each generalized symmetry of a gauge system gives rise to a sequence of conservation laws that are represented by onshell closed forms of various degrees. This extends the usual Noether's correspondence between global symmetries and conservation laws to the case of lower-degree conservation laws and not necessarily variational equations of motion. Finally, we equip the space of conservation laws of a given degree with a Lie bracket and establish a homomorphism of the resulting Lie algebra to the Lie algebra of global symmetries.
\end{abstract}

Key words: variational bicomplex; BRST differential; presymplectic structure; lower-degree conservation laws

2010 Mathematics Subject Classification: 70S10; 81T70; 83C40

\section{Introduction}

In this paper, we continue our study of the variational tricomplex and its applications initiated in [27]. Loosely, the variational tricomplex may be viewed as the standard variational bicomplex $[2,11,24,26]$ endowed with one more coboundary operator, namely, the classical BRST differential. The BRST differential carries an exhaustive information about the classical equations of motion, their gauge symmetries and identities. Although the BRST theory is commonly regarded as a tool for quantizing gauge theories [15], the classical BRST differential, as such, has nothing to do with quantization: to quantize a classical theory one or another extra structure is needed. In the context of the variational tricomplex such an extra ingredient is most naturally identified with a BRST-invariant presymplectic structure. Depending on the formalism one uses to describe classical dynamics, different kinds of objects can be identified as presymplectic structures. Within the Lagrangian formalism, for example, the presymplectic structure appears as an odd symplectic form underlying the BV bracket on the space of fields and antifields. In the BFV formalism of constrained Hamiltonian systems the same presymplectic structure reincarnates as the canonical symplectic structure on the extended phase space. As was shown in [27], the concept of variational tricomplex provides a uniform geometrical description of all these reincarnations, maintaining an explicit space-time covariance even in the Hamiltonian picture of dynamics. In particular, it allows one to pass directly from the BV to BFV formalism at the level of the BRST charge and master action; in so doing, the whole spectrum of BFV fields and the presymplectic structure are generated immediately from those of the BV theory. Moreover, with due definition of the BRST differential $[18,22]$ the concept of variational tricomplex extends beyond the scope of Lagrangian dynamics.

In the present paper, we focus upon the issues of global symmetries, conservation laws and interrelation between them. In mathematical terms the conservation laws are described by differential forms on an $n$-dimensional space-time manifold. The coefficients of these forms 
are assumed to be given by smooth functions of fields and their derivatives and the forms are required to be closed by virtue of equations of motion, that is, on-shell. Two conservation laws are considered as equivalent if they are represented by on-shell cohomologous differential forms. The degree of a conservation law is by definition the degree of a form it is represented by. Since the $n$-forms are automatically closed it makes sense to consider on-shell closed forms of degrees less or equal to $n-1$. These constitute the so-called characteristic cohomology of the system. The "ordinary" conservation laws have the maximal degree $n-1$, while those of degree $<n-1$ are usually referred to as the lower-degree conservation laws ${ }^{1}$. The typical example of the topdegree conservation law is provided by the energy-momentum tensor of the electromagnetic field, which in actuality represents four independent conserved quantities. Here we also encounter the lower-degree conservation law represented by the Hodge dual of the strength 2-form. The latter owes its existence to the gauge invariance of the electromagnetic potentials and expresses Gauss' law. Various results on lower-degree conservation laws, obtained by variety of techniques, can be found in $[5,8,14,29,30,31,32,33]$.

The notion of conservation law is closely related to the idea of symmetry. Indeed, each topdegree conservation law of a Lagrangian system defines and is defined by a global symmetry of the action functional. This is the precise content of Noether's first theorem on the link between symmetries and conservation laws [21]. The nature of the lower-degree conservation laws is somewhat different. As is well known [5], they owe their origin to (the special structure of) gauge symmetries, rather than to global invariance. The presence of gauge symmetries is thus a necessary but not a sufficient condition for the existence of lower-degree conservation laws. Due to the second Noether's theorem no ordinary conserved current corresponds to the gauge invariance of the action [21]. We have to conclude that the top- and lower-degree conservation laws are quite different things when viewed from the perspective of the conventional Lagrangian formalism.

This difference disappears entirely within the variational tricomplex approach, where the action functional is substituted by the classical BRST differential and the BRST-invariant presymplectic form. The global symmetries are then naturally identified with the infinitesimal transformations that leave invariant either structure. We show that each symmetry, being defined in such a way, gives rise to a sequence of conservation laws of decreasing degree. This allows us to treat the top- and lower-degree conservation laws on equal footing, i.e., as a manifestation of global symmetries. Furthermore, using the notion of a descendent presymplectic structure [27], we are able to endow the space of conservation laws of a given degree with a Lie bracket. In top-degree, this bracket reproduces the Dickey bracket in the space of conserved currents [11]. By construction, the Lie algebras of conservation laws come equipped with homomorphisms to the Lie algebra of original global symmetries and one may regard these homomorphisms as an extension of the first Noether's theorem to the case of lower-degree conservation laws. For Lagrangian gauge systems in the BV-BRST formalism such a connection between higher symmetries and lower-degree conservation laws was established in [5].

Unification of top- and lower-degree conservation laws is not the only advantage of our approach. The chief value of the concept of variational tricomplex is that it equally well applies to non-Lagrangian theories. In general, the existence of a compatible presymplectic structure imposes less restrictions on the classical dynamics than the existence of a Lagrangian. Among recent examples of this kind let us mention the derivation of conserved currents for the nonLagrangian equations of motion governing the dynamics of massless higher-spin fields [28]. It should be noted that one and the same system of classical equations of motion may admit, in

\footnotetext{
${ }^{1}$ In physics it is customary to describe the conservation laws by polyvectors rather than forms. Since the passage from forms to polyvectors involves the Hodge dualization w.r.t. to some background metric, the higher the form-degree, the lower the polyvector-degree and vice versa. Correspondingly, the lower-degree conservation laws from the viewpoint of forms become of higher-degree in terms of polyvectors.
} 
principle, several inequivalent presymplectic structures. Not only do these presymplectic structures lead to different quantizations, but they also lead to different links between symmetries and conservation laws in the classical theory. It might be well to point out in this connection that another generalization of the first Noether's theorem to non-Lagrangian gauge theories was proposed in [16, 17].

The paper is organized as follows. In the next section, we review the concepts of variational tricomplex and presymplectic structure. Here we also recall the notion of a descendent gauge system [27], which is basic to our subsequent considerations. In Section 3, we introduce and study the notions of physical observables, (Hamiltonian) symmetries and conservation laws. Among other thing we show that each Hamiltonian symmetry originates from a physical observable and the latter gives rise to a sequence of conservation laws of various degrees. In Section 4, we slightly relax the defining conditions for a Hamiltonian symmetry and this enables us to endow the space of conservation laws with the structure of a Lie algebra. The corresponding Lie bracket is determined by the descendent presymplectic structure. In Section 5, the general formalism is illustrated by three examples of physical interest: Maxwell's electrodynamics, the Chern-Simons theory, and the linearized gravity in the vierbein formalism. Appendix A contains some basic facts concerning the geometry of jet bundles and the variational bicomplex.

\section{Variational tricomplex of a local gauge system}

Let $M$ be an $n$-dimensional space-time manifold. In modern language the classical fields are just the sections of a locally trivial fiber bundle $\pi: E \rightarrow M$. The typical fiber $F$ of $E$ is called the target space of fields. For trivial bundles, $E=M \times F$, the fields are merely smooth mappings $\phi: M \rightarrow F$. For simplicity we restrict ourselves to vector bundles, in which case the space of fields $\Gamma(E)$ has the structure of vector space. At the same time, to accommodate fermionic fields as well as ghost fields associated with gauge symmetries, we assume $\pi: E \rightarrow M$ to be a $\mathbb{Z}$-graded supervector bundle. This means that the typical fiber $F$ has the structure of a $\mathbb{Z}$ graded superspace, while the base $M$ remains an ordinary (nongraded) manifold $M$. Following the physical tradition, we refer to the $\mathbb{Z}$-grading as the ghost number and denote the degree of a homogeneous object $A$ by $\operatorname{gh}(A)$. The Grassmann parity will be denoted by $\epsilon(A)$. The latter is responsible for the sign rule. It should be emphasized that in the presence of fermions there is no natural correlation between the Grassmann parity and the ghost number. Since throughout the paper we work exclusively in the category of $\mathbb{Z}$-graded supervector bundles, we omit the boring prefixes "super" and "graded" whenever possible. For a quick introduction to the graded differential geometry we refer the reader to [9, 23, 25, 34].

A fundamental tenet of classical field theory is locality. Above all it implies that the dynamics of fields are governed by partial differential equations. The basic tool for a geometric approach to differential equations is provided by the jet bundle formalism. In our case a relevant jet bundle is the bundle $\pi_{\infty}: J^{\infty} E \rightarrow M$ of infinite jets associated with the vector bundle $\pi: E \rightarrow M$. The differential forms on $J^{\infty} E$ carry the structure of double complex. This double complex is called the variational bicomplex because one of its differentials coincides with the variational derivative. This leads one to a formal variational calculus that can be viewed as a geometrized version of the classical calculus of variations. The free variational bicomplex represents thus a natural kinematical basis for formulating local field theories. It is summarized in Appendix A, where we also explain our notation. In the recent paper [27], the concept of variational bicomplex was enhanced by introducing two more geometrical ingredients: a classical BRST differential and a BRST-invariant presymplectic structure. The former brings dynamics into the free variational bicomplex by making it into a tricomplex, while the latter is responsible for quantization and, as we will show below, for establishing a correspondence between symmetries and conservation laws. Let us describe these two extra structures in more detail. 


\subsection{Presymplectic structure}

By a presymplectic $(2, m)$-form on $J^{\infty} E$ we understand an element $\omega \in \Lambda^{2, m}\left(J^{\infty} E\right)$ satisfying

$$
\delta \omega \simeq 0 .
$$

The sign $\simeq$ means equality modulo $d$-exact forms. It might be worth to mention that the horizontal degree $m$ of the presymplectic form need not be a priori related to the dimension $n$ of the space-time manifold $M$. Two presymplectic forms are considered as equivalent if they differ by a $d$-exact form. In what follows, we will not distinguish between $\omega \in \Lambda^{2, m}\left(J^{\infty} E\right)$ and its equivalence class $[\omega]$ in the quotient space $\widetilde{\Lambda}^{2, m}\left(J^{\infty} E\right)=\Lambda^{2, m}\left(J^{\infty} E\right) / d \Lambda^{2, m-1}\left(J^{\infty} E\right)$, denoting both by $\omega$. According to the definition above the presymplectic forms are the cocycles of the relative " $\delta$ modulo $d$ " cohomology in vertical degree 2 .

The form $\omega$ is assumed to be homogeneous, so that we can speak of an odd or even presymplectic structure of definite ghost number. The triviality of the relative " $\delta$ modulo $d$ " cohomology ${ }^{2}$ in positive vertical degree (see [11, Section 19.3.9]) implies that any presymplectic $(2, m$ )-form is exact, namely, there exists a homogeneous $(1, m)$-form $\theta$ such that $\omega \simeq \delta \theta$. The form $\theta$ is called a presymplectic potential for $\omega$. The presymplectic potential is obviously not unique. If $\theta_{0}$ is one of the presymplectic potentials for $\omega$, then setting $\omega_{0}=\delta \theta_{0}$ we get

$$
\delta \omega_{0}=0, \quad \omega_{0} \simeq \omega .
$$

In other words, any presymplectic form has a $\delta$-closed representative.

An evolutionary vector field $X$ is called Hamiltonian with respect to $\omega$ if it preserves the presymplectic form, that is,

$$
L_{X} \omega \simeq 0
$$

Obviously, the Hamiltonian vector fields form a subalgebra in the Lie algebra of all evolutionary vector fields. We denote this subalgebra by $\mathfrak{X}_{\omega}\left(J^{\infty} E\right)$. Equation (2.1) is equivalent to

$$
\delta i_{X} \omega \simeq 0 .
$$

Again, because of the triviality of the relative $\delta$-cohomology, we can write

$$
i_{X} \omega \simeq \delta H
$$

for some $H \in \Lambda^{0, m}\left(J^{\infty} E\right)$. We refer to $H$ as a Hamiltonian form (or Hamiltonian) associated with $X$. It is clear that equation (2.2) defines the Hamiltonian only modulo adding to $H$ a $d$-exact form. Therefore, two Hamiltonian forms $H$ and $H^{\prime}$ will be considered as equivalent if $H \simeq H^{\prime}$. By abuse of notation, we will use the same symbol $H$ to denote a particular Hamiltonian form and its equivalence class. Sometimes, to indicate the relation between the Hamiltonian vector fields and forms, we will write $X_{H}$ for $X$. In general, this relationship is far from being one-to-one.

The space $\Lambda_{\omega}^{m}\left(J^{\infty} E\right)$ of all Hamiltonian $m$-forms can be endowed with the structure of a Lie algebra. The corresponding Lie bracket is defined as follows: If $X_{A}$ and $X_{B}$ are two Hamiltonian vector fields associated with the Hamiltonian forms $A$ and $B$, then

$$
\{A, B\}=(-1)^{\epsilon\left(X_{A}\right)} i_{X_{A}} i_{X_{B}} \omega .
$$

The next proposition shows that the bracket is well defined and possesses all the required properties.

\footnotetext{
${ }^{2}$ Recall that we have restricted ourselves to the fields associated with vector bundles, where the target space of fields is contractible. For more general fiber bundles the triviality of the relative $\delta$-cohomology should be taken as hypothesis.
} 
Proposition 2.1 ([27]). The bracket (2.3) is bilinear over reals, maps the Hamiltonian forms to Hamiltonian ones, enjoys the symmetry property

$$
\{A, B\} \simeq-(-1)^{(\epsilon(A)+\epsilon(\omega))(\epsilon(B)+\epsilon(\omega))}\{B, A\},
$$

and obeys the Jacobi identity

$$
\{C,\{A, B\}\} \simeq\{\{C, A\}, B\}+(-1)^{(\epsilon(C)+\epsilon(\omega))(\epsilon(A)+\epsilon(\omega))}\{A,\{C, B\}\} .
$$

Combining equations (2.2) and (2.3), one can see that

$$
\{A, B\} \simeq(-1)^{\epsilon(A)} L_{X_{A}} B .
$$

The last relation gives an equivalent definition for the Poison bracket.

Let $\operatorname{ker} \omega$ denote the space of all Hamiltonian vector fields $X$ with zero Hamiltonian, i.e.,

$$
i_{X} \omega \simeq 0
$$

It is easy to see that $\operatorname{ker} \omega$ is an ideal in the Lie algebra of Hamiltonian vector fields $\mathfrak{X}_{\omega}\left(J^{\infty} E\right)$. One can regard the quotient $\mathfrak{X}_{\omega}\left(J^{\infty} E\right) /$ ker $\omega$ as the Lie algebra of nontrivial Hamiltonian vector fields. The next proposition relates this Lie algebra to the Lie algebra of Hamiltonian forms.

Proposition 2.2. There is a short exact sequence

$$
0 \longrightarrow \Lambda^{m}(M) \stackrel{\pi_{\infty}^{*}}{\longrightarrow} \Lambda_{\omega}^{m}\left(J^{\infty} E\right) \stackrel{\alpha}{\longrightarrow} \mathfrak{X}_{\omega}\left(J^{\infty} E\right) / \operatorname{ker} \omega \longrightarrow 0
$$

where $\pi_{\infty}^{*}$ is the pull back of the canonical projection $\pi_{\infty}: J^{\infty} E \rightarrow M$ and the map $\alpha$ assigns to each Hamiltonian form $A$ the equivalence class $X_{A}+\operatorname{ker} \omega$.

We leave it to the reader to check exactness. It is significant that $\alpha$ is a homomorphism of Lie algebras [27]. This means that

$$
X_{\{A, B\}}=\left[X_{A}, X_{B}\right] \quad(\bmod \quad \operatorname{ker} \omega) \quad \forall A, B \in \Lambda_{\omega}^{m}\left(J^{\infty} E\right),
$$

and the ideal ker $\alpha$ consists of the field-independent differential forms.

It follows from the definition (2.2) that each Hamiltonian form is necessarily invariant w.r.t. the action of the kernel distribution, that is,

$$
L_{X} A \simeq 0 \quad \forall X \in \operatorname{ker} \omega, \quad \forall A \in \Lambda_{\omega}^{m}\left(J^{\infty} E\right) .
$$

Therefore, the more degenerate the presymplectic structure, the less the size of the space of Hamiltonian forms. A presymplectic form $\omega$ is called nondegenerate if $\operatorname{ker} \omega=0$, in which case we refer to it as a symplectic form.

For a general discussion of a presymplectic structure as well as numerous applications of this notion in field theory we refer the reader to the papers $[1,7,10,12,19,20,28,35]$ and the references therein.

\subsection{Classical BRST differential}

An odd evolutionary vector field $Q$ on $J^{\infty} E$ is called homological if

$$
[Q, Q]=2 Q^{2}=0, \quad \operatorname{gh}(Q)=1 .
$$

We will use the special notation $\delta_{Q}$ for the Lie derivative along the homological vector field $Q$. It follows from the definition that $\delta_{Q}^{2}=0$. Hence, $\delta_{Q}$ is a differential of the algebra $\Lambda^{*, *}\left(J^{\infty} E\right)$ 
increasing the ghost number by 1 . Moreover, the operator $\delta_{Q}$ anticommutes with the coboundary operators $d$ and $\delta$ :

$$
\delta_{Q} d+d \delta_{Q}=0, \quad \delta_{Q} \delta+\delta \delta_{Q}=0
$$

This allows us to speak of the tricomplex $\Lambda^{*, * *}\left(J^{\infty} E ; \delta, d, \delta_{Q}\right)$, where

$$
\delta_{Q}: \Lambda^{p, q, r}\left(J^{\infty} E\right) \rightarrow \Lambda^{p, q, r+1}\left(J^{\infty} E\right)
$$

and $r$ is the ghost number.

In the physical literature the operator $\delta_{Q}$ is called the classical BRST differential and we will also use this term to refer to the homological vector field $Q$ itself.

The equations of motion of a gauge theory are recovered by considering the zero locus of $Q$. In terms of the adapted coordinates $\left(x^{i}, \phi_{I}^{a}\right)$ on $J^{\infty} E$ the vector field $Q$, being evolutionary, assumes the form ${ }^{3}$

$$
Q=\partial_{I} Q^{a} \frac{\partial}{\partial \phi_{I}^{a}}
$$

Then there exists an integer $l$ such that the equations

$$
\partial_{I} Q^{a}=0, \quad|I|=k,
$$

define a submanifold $\Sigma^{k} \subset J^{l+k} E$. The standard regularity condition implies that $\Sigma^{k+1}$ fibers over $\Sigma^{k}$ for each $k$. This gives the infinite sequence of projections

$$
\cdots \longrightarrow \Sigma^{l+3} \longrightarrow \Sigma^{l+2} \longrightarrow \Sigma^{l+1} \longrightarrow \Sigma^{l} \longrightarrow M,
$$

which enables us to define the zero locus of $Q$ as the inverse limit

$$
\Sigma^{\infty}=\lim _{\longleftarrow} \Sigma^{k}
$$

In physics, the submanifold $\Sigma^{\infty} \subset J^{\infty} E$ is usually referred to as the shell. The terminology is justified by the fact that the classical field equations as well as their differential consequences can be written as $^{4}$

$$
\left(j^{\infty} \phi\right)^{*}\left(\partial_{I} Q^{a}\right)=0
$$

In other words, the field $\phi \in \Gamma(E)$ satisfies the classical equations of motion iff $j^{\infty} \phi \in \Sigma^{\infty}$. In the conventional BRST theory of variational gauge systems, the relationship between the zero locus of the classical BRST differential and solutions to the classical equations of motion was studied in [13]. The extension to non-Lagrangian gauge systems may be found in [18].

It follows from (2.6) that the shell $\Sigma^{\infty}$ is invariant under the action of $Q$. This makes possible to pull the "free" variational tricomplex $\Lambda^{*, * *}\left(J^{\infty} E ; \delta, d, \delta_{Q}\right)$ back to $\Sigma^{\infty}$ and so define the on-shell tricomplex $\Lambda^{*, * *}\left(\Sigma^{\infty} ; \delta, d, \delta_{Q}\right)$. The latter is not generally $d$-exact even locally and this gives rise to various interesting cohomology groups associated with gauge dynamics. For example, the groups $H^{0, *, 0}\left(\Sigma^{\infty} ; d\right)$ describe the so-called characteristic cohomology of a gauge

\footnotetext{
${ }^{3}$ We use the multi-index notation according to which the multi-index $I=i_{1} i_{2} \cdots i_{k}$ represents the set of symmetric covariant indices and $\partial_{I}=\partial_{i_{1}} \cdots \partial_{i_{k}}$. The order of the multi-index is given by $|I|=k$.

${ }^{4}$ To avoid any confusion, let us stress that the collection of fields $\phi$ includes both the "usual fields" (i.e., those with ghost number zero) and the ghost fields. Accordingly, by the classical field equations we mean partial differential equations for the whole collection of fields $\phi$. The equations for "usual fields" are then obtained by projecting the shell to the sector of ghost number zero.
} 
system, see $[5,8,14,29,30,31,32,33]$ and Section 3 below. The interpretation of some other groups can be found in [17].

It should be noted that the first variational tricomplex for gauge systems was introduced in [6] as the Koszul-Tate resolution of the usual variational bicomplex for partial differential equations. Using this tricomplex, the authors of [6] were able to relate various Lie algebras associated with the global symmetries and conservation laws of a classical gauge system. Our tricomplex is similar in nature but involves the full BRST differential, and not its Koszul-Tate part.

\section{3 $Q$-invariant presymplectic structure and its descendants}

By a gauge system on $J^{\infty} E$ we mean a pair $(Q, \omega)$ consisting of a classical BRST differential $Q$ and a $Q$-invariant presymplectic $(2, m)$-form $\omega$. In other words, the vector field $Q$ is supposed to be Hamiltonian with respect to $\omega$, so that $\delta_{Q} \omega \simeq 0$. Then, according to (2.1) and (2.2), there exist forms $\omega_{1}, L$, and $\theta_{1}$ such that

$$
\delta_{Q} \omega=d \omega_{1}, \quad i_{Q} \omega=\delta L+d \theta_{1},
$$

with $L$ being the Hamiltonian for $Q$ relative to $\omega$. As was mentioned in Section 2.1, we can always assume that $\omega=\delta \theta$ for some presymplectic potential $\theta$, so that $\delta \omega=0$. Then applying $\delta$ to the second equality in (2.8) and using the first one, we find $d\left(\omega_{1}-\delta \theta_{1}\right)=0$. On account of the exactness of the variational bicomplex the last relation is equivalent to

$$
\omega_{1} \simeq \delta \theta_{1} .
$$

Thus, $\omega_{1}$ is a presymplectic $(2, m-1)$-form on $J^{\infty} E$ with the presymplectic potential $\theta_{1}$. Furthermore, the form $\omega_{1}$ is $Q$-invariant as one can easily see by applying $\delta_{Q}$ to the first equality in (2.8) and using once again the fact of exactness of the variational bicomplex. Let $L_{1}$ denote the Hamiltonian for $Q$ with respect to $\omega_{1}$, i.e.,

$$
i_{Q} \omega_{1} \simeq \delta L_{1}, \quad L_{1} \in \Lambda^{0, m-1}\left(J^{\infty} E\right) .
$$

Given the pair $(Q, \omega)$, we call $\omega_{1}$ the descendent presymplectic structure on $J^{\infty} E$ and refer to $\left(Q, \omega_{1}\right)$ as the descendent gauge system. This construction of a descendent gauge system can be iterated producing a sequence of gauge systems $\left(Q, \omega_{k}\right)$, where the $k$-th presymplectic form $\omega_{k} \in \Lambda^{2, m-k}\left(J^{\infty} E\right)$ is the descendant of the previous form $\omega_{k-1}$. The minimal $k$ for which $\omega_{k} \simeq 0$ gives a numerical invariant of the original gauge system $(Q, \omega)$. We call it the length of a gauge system.

\section{Symmetries, observables and conservation laws}

Definition 3.1. Given a classical BRST differential $Q$, a form $\alpha \in \Lambda^{0, m}\left(J^{\infty} E\right)$ is said to define a conservation law of degree $m$ if

$$
\left.d \alpha\right|_{\Sigma \infty}=0
$$

The conservation law is called trivial if $\left.\alpha\right|_{\Sigma^{\infty}} \simeq 0$.

In other words, the conservation laws are represented by the on-shell closed forms and the trivial conservation laws correspond to the on-shell exact forms. This allows us to identify the space of nontrivial conservation laws with the cohomology groups $H^{0, m}\left(\Sigma^{\infty} ; d\right)$ of the on-shell variational bicomplex. In addition to the form degree these groups are also graded by the ghost 
number. In what follows, the form degree of a horizontal form $\alpha \in \Lambda^{0, m}\left(J^{\infty} E\right)$ will be denoted by $\operatorname{deg} \alpha=m$.

Due to the standard regularity condition [5, Section 5.1], equation (3.1) implies the existence of a form $\chi$ such that

$$
d \alpha=i_{Q} \chi
$$

The form $\chi$ is called the characteristic of the conservation law $\alpha$. Note that shifting a characteristic by a $d$-exact form one does not change the equivalence class of the corresponding conservation law. This gives a natural equivalence relation on the space of characteristics. A characteristic $\chi$ is called trivial if $\chi \simeq 0$.

Given a conservation law represented by an $m$-form $\alpha$ together with an $m$-cycle $C \subset M$ and a field configuration $\phi \in \Gamma(E)$, we can define the integral

$$
I[\phi]=\int_{C}\left(j^{\infty} \phi\right)^{*}(\alpha) .
$$

By construction, the integrand is given by a closed form on $M$ provided that $j^{\infty} \phi \in \Sigma^{\infty}$. Therefore, for a fixed solution $\phi$, the value of the integral depends only on the homology class of $C$ in $M$. It is the invariance of the functional $I[\phi]$ under continues deformations of $C$ which is usually meant by a conservation law ${ }^{5}$. The functional $I[\phi]$ is called the conserved charge.

Definition 3.2. A form $\alpha \in \Lambda^{0, m}\left(J^{\infty} E\right)$ is called an observable of degree $m$ if

$$
\delta_{Q} \alpha \simeq 0
$$

An observable $\alpha$ is said to be trivial if $\alpha \simeq \delta_{Q} \beta$ for some $\beta$.

According to this definition the space of nontrivial observables of degree $m$ and ghost number $r$ is identified with the cohomology groups $H^{0, m, r}\left(J^{\infty} E ; \delta_{Q}\right)$. (Here we slightly deviate from the standard usage. Usually, by an observable in the BRST theory one means a $Q$-invariant quantity with ghost number zero, which corresponds to a gauge invariant local observable. According to our definition an observable may have nonzero ghost number.)

Proposition 3.3. Suppose that the complex

$$
0 \longrightarrow \mathbb{R} \longrightarrow \Lambda^{0,0}\left(J^{\infty} E\right) \stackrel{d}{\longrightarrow} \Lambda^{0,1}\left(J^{\infty} E\right) \stackrel{d}{\longrightarrow} \cdots \stackrel{d}{\longrightarrow} \Lambda^{0, n}\left(J^{\infty} E\right)
$$

is exact ${ }^{6}$. Then each observable $\alpha_{0}$ of degree $m$ gives rise to the sequence of observables and conservation laws $\left\{\alpha_{k}\right\}_{k=1}^{m}$, where the characteristic of $\alpha_{k}$ is given by $\delta \alpha_{k-1}$ and $\operatorname{deg} \alpha_{k}=m-k$. Trivial observables give rise to trivial conservation laws.

Note that the proposition does not assert that all conservation laws originating from a nontrivial observable are nontrivial.

Proof. We use the cohomological descent method [5]. From Definition 3.2 of an observable it follows that

$$
\delta_{Q} \alpha_{0}=d \alpha_{1}
$$

\footnotetext{
${ }^{5}$ In physical problems the $m$-chain $C$ is often noncompact (e.g., a time-slice in the Minkowski space), in which case some appropriate asymptotic conditions on the fields are imposed to provide the existence and conservation of the charge $I[\phi]$.

${ }^{6}$ This is the case, for example, when $M \simeq \mathbb{R}^{n}$.
} 
for some $\alpha_{1}$ of degree $m-1$. By Definition 3.1, $\alpha_{1}$ is a conservation law with characteristic $\delta \alpha_{0}$. Applying the differential $\delta_{Q}$ to both sides of (3.3) yields $d \delta_{Q} \alpha_{1}=0$. The complex (3.2) being exact, we can write $\delta_{Q} \alpha_{1}=d \alpha_{2}$ for some $\alpha_{2} \in \Lambda^{0, m-2}\left(J^{\infty} E\right)$. Thus, $\alpha_{1}$ is an observable and $\alpha_{2}$ is a conservation law with characteristic $\delta \alpha_{1}$. Iterating this construction once and again, we get the sequence $\left\{\alpha_{k}\right\}$ of observables and conservation laws.

If $\alpha_{0}$ is a trivial observable, then $\alpha_{0}=\delta_{Q} \beta+d \gamma$ and $\alpha_{1}=\delta_{Q} \gamma+d \sigma$ for some $\sigma$. Hence, $\alpha_{1}$ is trivial as an observable and as a conservation law.

Definition 3.4. An evolutionary vector field $X$ is called a symmetry of a gauge system if it preserves the classical BRST differential, that is,

$$
[X, Q]=0 .
$$

It follows from the definition that the flow generated by $X$ preserves the shell $\Sigma^{\infty}$ mapping solutions to solutions.

Definition 3.5. A symmetry $X$ is called trivial or a gauge symmetry, if there exists another evolutionary vector field $Y$ such that $X=[Q, Y]$.

It is easy to see that the gauge symmetries form an ideal $\mathfrak{X}_{\mathrm{GS}}\left(J^{\infty} E\right)$ in the Lie algebra of all symmetries $\mathfrak{X}_{S}\left(J^{\infty} E\right)$. Therefore, it is natural to identify the Lie algebra of nontrivial symmetries with the quotient $\mathfrak{X}_{S}\left(J^{\infty} E\right) / \mathfrak{X}_{\mathrm{GS}}\left(J^{\infty} E\right)$. The latter can also be regarded as the group of $\delta_{Q}$-cohomology, with the differential $\delta_{Q}$ - the Lie derivative along $Q$ - acting in the space of evolutionary vector fields.

In this paper, we are mostly interested in the Hamiltonian symmetries of gauge systems.

Definition 3.6. A symmetry $X$ is called Hamiltonian if $X$ is a Hamiltonian vector field.

Proposition 3.7. The Hamiltonian of a Hamiltonian symmetry is an observable. Trivial Hamiltonian symmetries corresponds to trivial observables.

Proof. By definition we have

$$
i_{X} \omega \simeq \delta \alpha,
$$

where $\alpha$ is a Hamiltonian of $X$. Acting by $\delta_{Q}$ on both the sides of the last expression, we get

$$
\delta \delta_{Q} \alpha \simeq 0 .
$$

By Proposition A.1,

$$
\delta_{Q} \alpha=\pi_{\infty}^{*}(\beta)+d \gamma
$$

where $\beta$ is a differential form on $M$. If $\operatorname{gh}(\beta)=\operatorname{gh}(\alpha)+1 \neq 0$, then automatically $\beta=0$ as we have no parameters with nonzero ghost number. In the general case, consider a solution $\phi \in \Gamma(E)$ to the equations of motion (2.7). We have $\left(j^{\infty} \phi\right)^{*}\left(\delta_{Q} \alpha\right)=\left(j^{\infty} \phi\right)^{*}\left(i_{Q} \delta \phi\right)=0$. Applying now the pullback $\left(j^{\infty} \phi\right)^{*}$ to both the sides of (3.4), we find $\beta=-d\left(j^{\infty} \phi\right)^{*}(\gamma)$. Hence, $\delta_{Q} \alpha \simeq 0$ and the form $\alpha$ is an observable.

If $X=\delta_{Q} Y$, then, according to (2.4) and (2.5), the Hamiltonian of $X$ is given by the form $\alpha=-\delta_{Q} \beta+d \gamma$, where $\beta$ is the Hamiltonian of $Y$ and $\gamma$ is an arbitrary $(m-1)$-form. Thus, $\alpha$ is a trivial observable.

Combining the last proposition with Proposition 3.3, we arrive at the following statement.

Corollary 3.8. If the sequence (3.2) is exact, then each Hamiltonian symmetry gives rise to a sequence of conservation laws, perhaps trivial. 
For example, the classical BRST differential $Q$ can be viewed as a symmetry for itself. So, it gives rise to a conservation law $L_{1}$ defined by the equation $\delta_{Q} L=d L_{1}$, where $L$ is the Hamiltonian of $Q$ relative to $\omega$. It is not hard to see [27] that the form $L_{1}^{\prime}=L_{1}+i_{Q} \theta_{1}$, defining an equivalent conservation law, is Hamiltonian relative to the descendent presymplectic structure $\omega_{1}=\delta \theta_{1}$. Indeed, applying $\delta_{Q}$ to both the sides of the second equality in (2.8), we get

$$
\begin{aligned}
& i_{Q} \delta_{Q} \omega=-\delta \delta_{Q} L-d \delta_{Q} \theta_{1}, \\
& i_{Q} d \omega_{1}=-\delta d L_{1}-d\left(i_{Q} \delta-\delta i_{Q}\right) \theta_{1}, \\
& d i_{Q} \omega_{1}=d \delta\left(L_{1}+i_{Q} \theta_{1}\right)-d i_{Q} \omega_{1},
\end{aligned}
$$

and hence

$$
2 i_{Q} \omega_{1} \simeq \delta\left(L_{1}+i_{Q} \theta_{1}\right)=\delta L_{1}^{\prime} .
$$

It then follows from equation (2.5) that $\delta\left\{L_{1}^{\prime}, L_{1}^{\prime}\right\} \simeq 8 i_{Q^{2}} \omega_{1}=0$. If $\operatorname{gh}\left\{L_{1}^{\prime}, L_{1}^{\prime}\right\} \neq 0$, then

$$
\left\{L_{1}^{\prime}, L_{1}^{\prime}\right\}_{1} \simeq 0
$$

In the case where $L$ is a form of top horizontal degree, the integral

$$
\Omega[\phi]=\int_{N}\left(j^{\infty} \phi\right)^{*}\left(L_{1}^{\prime}\right)
$$

over a Cauchy hypersurface $N \subset M$ is called the classical BRST charge and equation (3.5) is known as the classical master equation, see [27].

In a sense the example of the BRST symmetry $Q$ is the exception rather than the rule. Generally the conservation laws associated with Hamiltonian symmetries are neither Hamiltonian nor equivalent to Hamiltonian (relative to the descendent presymplectic structure). In the next section, we will see that the descendent presymplectic forms do induce appropriate Lie brackets on the conservation laws of various degrees providing one properly extends the notion of a Hamiltonian form.

\section{The Lie algebra of conservation laws}

As was mentioned in Section 2.2 the variational tricomplex admits a consistent restriction to the shell $\Sigma^{\infty}$. The cochains of the on-shell tricomplex can be identified with the equivalence classes of differential forms on $J^{\infty} E$, where two forms $\alpha$ and $\beta$ are considered equivalent if

$$
\left.\alpha\right|_{\Sigma^{\infty}}=\left.\beta\right|_{\Sigma^{\infty}}
$$

For the further convenience we also introduce the sign of "weak equality" $\approx$ meaning that

$$
\left.\left.\alpha \approx \beta \quad \Leftrightarrow \quad \alpha\right|_{\Sigma^{\infty}} \simeq \beta\right|_{\Sigma^{\infty}}
$$

Due to the regularity condition for $\Sigma^{\infty}$, the equation $\alpha \approx 0$ simply means that there exists a $d$-exact form $d \sigma$ such that the difference $\alpha-d \sigma$ belongs to the differential ideal of $\Lambda^{*, *}\left(J^{\infty} E\right)$ algebraically generated by all the differential forms of the form $i_{Q} \beta$ and $\delta_{Q} \gamma$.

Definition 4.1. A symmetry $X$ of a gauge system $(Q, \omega)$ is called on-shell Hamiltonian if there exists a form $\alpha$ such that

$$
i_{X} \omega \approx \delta \alpha .
$$


As a consequence of the definition, $L_{X} \omega \approx 0$ for any on-shell Hamiltonian symmetry $X$. The converse is not always true as the on-shell bicomplex may not be globally exact in columns even if the underlying fiber bundle of fields $\pi: E \rightarrow M$ is a vector bundle. It is obvious that the Hamiltonian symmetries form a subalgebra in the Lie algebra of all on-shell Hamiltonian symmetries. We denote the latter by $\mathfrak{X}_{\omega, Q}\left(J^{\infty} E\right)$.

Equation (4.1) defines $\alpha$ only modulo $d$-exact and on-shell vanishing forms. A form $\alpha$ satisfying (4.1) for some symmetry $X$ will be called on-shell Hamiltonian. Two on-shell Hamiltonian forms $\alpha$ and $\alpha^{\prime}$ associated with one and the same symmetry $X$ will be considered as equivalent if $\alpha^{\prime} \approx \alpha$. Due to the regularity of the shell the last equality is equivalent to the existence of forms $\beta$ and $\gamma$ such that $\alpha^{\prime}-\alpha=i_{Q} \beta+d \gamma$.

Proposition 4.2. The equivalence classes of on-shell Hamiltonian forms make a Lie algebra with respect to the bracket

$$
\{\alpha, \beta\}=(-1)^{\epsilon(X)} i_{X} i_{Y} \omega
$$

where $X$ and $Y$ are symmetries associated with $\alpha$ and $\beta$, respectively.

The proof of this proposition literally repeats that of Proposition 2.1 if one replaces the equality $\simeq$ by the weaker one $\approx$. The Lie algebra of all on-shell Hamiltonian $m$-forms will be denoted by $\Lambda_{\omega, Q}^{m}\left(J^{\infty} E\right)$. For Lagrangian theories without gauge symmetries the Lie bracket (4.2) of the first integrals of motion was studied in [11].

Let $\operatorname{ker}_{Q} \omega$ denote the space of all symmetries satisfying the homogeneous equation

$$
i_{X} \omega \approx 0 \text {. }
$$

It is clear that $\operatorname{ker}_{Q} \omega$ contains the intersection $\mathfrak{X}_{S}\left(J^{\infty} E\right) \cap \operatorname{ker} \omega$ and is contained in $\mathfrak{X}_{\omega, Q}\left(J^{\infty} E\right)$. If now $X \in \operatorname{ker}_{Q} \omega$ and $Y \in \mathfrak{X}_{\omega, Q}\left(J^{\infty} E\right)$, then

$$
L_{Y} i_{X} \omega \approx i_{[Y, X]} \omega \approx 0
$$

This shows that $\operatorname{ker}_{Q} \omega$ is an ideal of the Lie algebra $\mathfrak{X}_{\omega, Q}\left(J^{\infty} E\right)$ and we can define the quotient algebra $\mathfrak{X}_{\omega, Q}\left(J^{\infty} E\right) / \operatorname{ker}_{Q} \omega$.

Proposition 4.3. The correspondence $\alpha \mapsto X_{\alpha}+\operatorname{ker}_{Q} \omega$ defines a homomorphism

$$
f: \Lambda_{\omega, Q}^{m}\left(J^{\infty} E\right) \rightarrow \mathfrak{X}_{\omega, Q}\left(J^{\infty} E\right) / \operatorname{ker}_{Q} \omega
$$

of the Lie algebras.

The proof is straightforward. Notice that $\operatorname{ker} f$ contains the field-independent forms, i.e., the elements of im $\pi_{\infty}^{*}$. Belonging to the center of the Lie algebra $\Lambda_{\omega, Q}^{m}\left(J^{\infty} E\right)$, these forms are responsible for appearance of possible central charges in the Lie algebra of on-shell Hamiltonian symmetries or, more properly, in its preimage in $\Lambda_{\omega, Q}^{m}\left(J^{\infty} E\right)$.

Theorem 4.4. Let $\left\{\alpha_{k}\right\}$ be the sequence of conservation laws associated with a Hamiltonian symmetry $X$. Then the form $\alpha_{k}$ is on-shell Hamiltonian w.r.t. the $k$-th descendent presymplectic structure.

Proof. Let $\alpha$ be a Hamiltonian of $X$. Then

$$
i_{X} \omega=\delta \alpha+d \alpha^{\prime}
$$


form some $\alpha^{\prime}$. Applying $\delta_{Q}$ to the last equality, we get

$$
\begin{aligned}
& -(-1)^{\epsilon(X)} i_{X} \delta_{Q} \omega=-(-1)^{\epsilon(X)} \delta \delta_{Q} \alpha-d \delta_{Q} \alpha^{\prime}, \\
& -(-1)^{\epsilon(X)} i_{X} d \omega_{1}=-(-1)^{\epsilon(X)} \delta d \alpha_{1}-d \delta_{Q} \alpha^{\prime}, \\
& d i_{X} \omega_{1}=d \delta \alpha_{1}+d \delta_{Q} \alpha^{\prime} .
\end{aligned}
$$

This implies

$$
i_{X} \omega_{1}=\delta \alpha_{1}+\delta_{Q} \alpha^{\prime}+d \alpha_{1}^{\prime}
$$

for some $\alpha_{1}^{\prime}$. Hence,

$$
i_{X} \omega_{1} \approx \delta \alpha_{1}
$$

and $\alpha_{1}$ is an on-shell Hamiltonian form relative to $\omega_{1}$. Now acting on both the sides of (4.4) by $\delta_{Q}$, we get

$$
-(-1)^{\epsilon(X)} i_{X} \delta_{Q} \omega_{1}=-(-1)^{\epsilon(X)} \delta \delta_{Q} \alpha_{1}-d \delta_{Q} \alpha_{1}^{\prime} .
$$

This relation coincides in form with the first line of (4.3). Therefore, there exists a form $\alpha_{2}^{\prime}$ such that

$$
i_{X} \omega_{2}=\delta \alpha_{2}+\delta_{Q} \alpha_{1}^{\prime}+d \alpha_{2}^{\prime}
$$

and we conclude that $\alpha_{2}$ is on-shell Hamiltonian. Iterating this construction once and again, we obtain the sequence of relations

$$
i_{X} \omega_{k}=\delta \alpha_{k}+\delta_{Q} \alpha_{k-1}^{\prime}+d \alpha_{k}^{\prime}
$$

meaning that all the forms $\alpha_{k}$ are on-shell Hamiltonian.

Combining the above theorem with Proposition 4.3, we arrive at

Corollary 4.5. The descendent conservation laws associated with Hamiltonian symmetries form Lie algebras w.r.t. the descendent Lie brackets.

In particular, if $\operatorname{ker}_{Q} \omega_{k}=0$, then the algebra $\Lambda_{Q, \omega_{k}}^{m-k}\left(J^{\infty} E\right)$ is given by a central extension of the Lie algebra $\mathfrak{X}_{\omega, Q}\left(J^{\infty} E\right)$. This statement may be viewed as a main result of the paper. It relates the conservation laws of various degrees to the symmetries of the gauge system $(Q, \omega)$, that is, to the evolutionary vector fields that preserve both the classical BRST differential $Q$ and the BRST invariant presymplectic structure $\omega$.

Given the sequence of conservation laws $\left\{\alpha_{k}\right\}$ associated with a Hamiltonian symmetry $X$, the minimal $k$ for which $\alpha_{k} \approx 0$ is called the length of the symmetry $X$.

\section{$5 \quad$ Some applications}

In this section, we illustrate the general formalism above by a few examples of physical interest. Since the gauge theories we are going to consider are originally formulated in terms of the Batalin-Vilkovisky formalism, we start with a brief explanation of how this formalism fits into our framework. For more details we refer the reader to [27]. 


\subsection{BV formalism}

The starting point of the BV formalism is an infinite-dimensional manifold $\mathcal{M}_{0}$ of gauge fields that live on an $n$-dimensional space-time $M$. Depending on a particular structure of gauge symmetry the manifold $\mathcal{M}_{0}$ is extended to an $\mathbb{N}$-graded manifold $\mathcal{M}$ containing $\mathcal{M}_{0}$ as its body. The new fields of positive $\mathbb{N}$-degree are called the ghosts and the $\mathbb{N}$-grading is referred to as the ghost number. Let us collectively denote all the original fields and ghosts by $\Phi^{A}$ and refer to them as fields. At the next step the space of fields $\mathcal{M}$ is further extended by introducing the odd cotangent bundle $\Pi T^{*}[-1] \mathcal{M}$. The fiber coordinates, called antifields, are denoted by $\Phi_{A}^{*}$. These are assigned with the following ghost numbers and Grassmann parities:

$$
\operatorname{gh}\left(\Phi_{A}^{*}\right)=-\operatorname{gh}\left(\Phi^{A}\right)-1, \quad \epsilon\left(\Phi_{A}^{*}\right)=\epsilon\left(\Phi^{A}\right)+1 \quad(\bmod 2) .
$$

Thus, the total space of the odd cotangent bundle $\Pi T^{*}[-1] \mathcal{M}$ becomes a $\mathbb{Z}$-graded supermanifold. The canonical Poisson structure on $\Pi T^{*}[-1] \mathcal{M}$ is determined by the following odd Poisson bracket in the space of smooth functionals of $\Phi$ and $\Phi^{*}$ :

$$
(A, B)=\int_{M}\left(\frac{\delta_{r} A}{\delta \Phi^{A}} \frac{\delta_{l} B}{\delta \Phi_{A}^{*}}-\frac{\delta_{r} A}{\delta \Phi_{A}^{*}} \frac{\delta_{l} B}{\delta \Phi^{A}}\right) d^{n} x .
$$

Here $d^{n} x$ is a volume form on $M$ and the subscripts $l$ and $r$ refer to the standard left and right functional derivatives. In the physical literature the above bracket is usually called the antibracket or the $B V$ bracket.

The functionals of the form

$$
A=\int_{M}\left(j^{\infty} \phi\right)^{*}(a)
$$

where $\phi=\left(\Phi, \Phi^{*}\right)$ and $a \in \widetilde{\Lambda}^{0, n}\left(J^{\infty} E\right)$, are called local. Under suitable boundary conditions for $\phi$ 's the map $a \mapsto A$ defines an isomorphism of vector spaces, which gives rise to a pulled-back Lie bracket on $\widetilde{\Lambda}^{0, n}\left(J^{\infty} E\right)$. This last bracket is determined by the symplectic structure

$$
\omega=\delta \Phi_{A}^{*} \wedge \delta \Phi^{A} \wedge d^{n} x
$$

on the jet bundle $J^{\infty} E$ of fields and antifields. By definition, $\operatorname{gh}(\omega)=-1$ and $\epsilon(\omega)=1$. We will denote this Lie bracket by the same round brackets.

The central goal of the BV formalism is the construction of a master action. This is given by a local functional

$$
S[\phi]=\int_{M}\left(j^{\infty} \phi\right)^{*}(L)
$$

obeying the classical master equation

$$
(S, S)=0 \quad \Leftrightarrow \quad\{L, L\} \simeq 0 .
$$

The master Lagrangian $L$ is required to be of ghost number zero and start with the Lagrangian $L_{0}$ of the original fields to which one couples vertices involving antifields. All these vertices can be found systematically from the master equation (5.2) by means of the homological perturbation theory [15].

Since the canonical symplectic structure (5.1) of the BV formalism is nondegenerate, any form of top horizontal degree is Hamiltonian, i.e., $\Lambda_{\omega}^{n}\left(J^{\infty} E\right)=\Lambda^{0, n}\left(J^{\infty} E\right)$. Then the action of the classical BRST differential on $\Lambda^{0, n}\left(J^{\infty} E\right)$ is canonically generated by the master Lagrangian:

$$
Q=(L, \cdot) .
$$


Because of the classical master equation (5.2), the operator $Q$ squares to zero. Fixing a volume form $d^{n} x$ on $M$ allows us to identify the spaces $\Lambda^{0, n}\left(J^{\infty} E\right)$ and $\Lambda^{0,0}\left(J^{\infty} E\right)$. Upon this identification the action (5.3) induces that in the space of 0 -forms. The latter specifies the evolutionary vector field $Q$ completely.

Thus, we see that the standard ingredients of the BV formalism - the antibracket and the master action - define a gauge system in our sense; in so doing, the classical BRST differential is generated by the master action through the antibracket.

The following statement is of particular importance for the BV formalism.

Proposition 5.1. Let $(Q, \omega)$ be a gauge system, with $\omega$ being a symplectic form. Then a Hamiltonian vector field $X_{A}$ with gh $A \neq-1$ is a symmetry iff it preserves the Hamiltonian of $Q$, i.e., $\mathcal{L}_{X_{A}} L \simeq 0$.

Proof. According to equations (2.4) and (2.5) we have

$$
\mathcal{L}_{X_{A}} L \simeq(-1)^{\epsilon\left(X_{A}\right)}\{A, L\} \quad \text { and } \quad i_{\left[X_{A}, Q\right]} \omega \simeq \delta\{A, L\} .
$$

If $X_{A}$ preserves $L$, then the r.h.s. of the second equation vanishes and we conclude that $\left[X_{A}, Q\right] \in$ $\operatorname{ker} \omega$. For symplectic forms this implies that $\left[X_{A}, Q\right]=0$; and hence, $X_{A}$ is a symmetry. Conversely, if $X_{A}$ is a symmetry, then the l.h.s. of the second equation vanishes and we get $\delta\{A, L\} \simeq 0$, where $\operatorname{gh}\{A, L\}=\operatorname{gh} A+1 \neq 0$. Since the relative $\delta$-cohomology is trivial in nonzero ghost number, we conclude that $\{A, L\} \simeq 0$. By the first equation, this means that $X_{A}$ preserves $L$.

Let us now turn to specific gauge systems.

\subsection{Maxwell's electrodynamics}

In the BV approach [15], the free electromagnetic field on a 4-dimensional space-time manifold $M$ is described by the gauge potential ${ }^{7} A \in \Lambda^{1}(M)$, the ghost field $C \in \Lambda^{0}(M)$ as well as their antifields $A^{*} \in \Lambda^{3}(M)$ and $C^{*} \in \Lambda^{4}(M)$. The ghost number distribution reads

$$
\operatorname{gh}\left(C^{*}\right)=-2, \quad \operatorname{gh}\left(A^{*}\right)=-1, \quad \operatorname{gh}(A)=0, \quad \operatorname{gh}(C)=1,
$$

and the Grassmann parity is just the ghost number modulo 2. The space of fields and antifields is endowed with the canonical symplectic structure

$$
\omega=\delta A \wedge \delta A^{*}+\delta C \wedge \delta C^{*}, \quad \operatorname{gh}(\omega)=-1 .
$$

The action of the classical BRST differential is given by the equations

$$
\delta_{Q} C^{*}=d A^{*}, \quad \delta_{Q} A^{*}=d \tilde{F}, \quad \delta_{Q} A=d C, \quad \delta_{Q} C=0 .
$$

Here $F=d A$ is the strength of the electromagnetic field and $\tilde{F}=* F$ is its Hodge dual. Notice that the Maxwell equations $d \tilde{F}=0$ are the part of the defining relations for the zero locus of $Q$. The Hamiltonian of the classical BRST differential $Q$ is given by the BV master Lagrangian

$$
L=\frac{1}{2} F \wedge \tilde{F}+A^{*} \wedge d C, \quad i_{Q} \omega \simeq \delta L .
$$

As a consequence of $Q^{2}=0$, the master Lagrangian $L$ satisfies the BV master equation

$$
\{L, L\}=-i_{Q}^{2} \omega \simeq 0 .
$$

\footnotetext{
${ }^{7}$ For the sake of simplicity, we assume that the gauge potential defines a connection in a trivial $U(1)$-bundle over $M$. This makes possible to identify the space of abelian connections with the space of 1-forms.
} 
Applying the BRST differential to (5.4) yields the descendent presymplectic structure

$$
\omega_{1}=\delta C \wedge \delta A^{*}+\delta A \wedge \delta \tilde{F}, \quad \delta_{Q} \omega=d \omega_{1}, \quad \operatorname{gh}\left(\omega_{1}\right)=0 .
$$

The descendent Hamiltonian of $Q$ is given by the conserved BRST current $L_{1}^{\prime}=2 L_{1}$, where

$$
L_{1}=C \wedge d \tilde{F}, \quad d L_{1}=\delta_{Q} L
$$

The current is obviously trivial as $L_{1} \approx 0$. Integrating $L_{1}^{\prime}$ over a Cauchy hypersurface $N \subset M$, we get the classical BRST charge $\Omega=\int_{N} L_{1}^{\prime}$. Again, in view of the equation $Q^{2}=0$, the BRST current obeys the classical master equation

$$
\left\{L_{1}^{\prime}, L_{1}^{\prime}\right\}_{1}=-i_{Q}^{2} \omega_{1} \simeq 0 .
$$

Acting now by the BRST differential on $\omega_{1}$, we get one more presymplectic structure of ghost number one

$$
\omega_{2}=\delta C \wedge \delta \tilde{F}, \quad \delta_{Q} \omega_{1}=d \omega_{2}, \quad \operatorname{gh}\left(\omega_{2}\right)=1 .
$$

This last form, being "absolutely" invariant under the BRST transformations (5.5), leaves no further descendants. Thus, the length of Maxwell's electrodynamics relative to the BV symplectic structure (5.4) equals 2.

Given a Killing vector $\xi$ of the background metric, one can define an even vector field $X$ on the space of fields and antifields. The latter is determined by the relations

$$
\delta_{X} \Phi=L_{\xi} \Phi, \quad \Phi=\left(A, C, A^{*}, C^{*}\right) .
$$

Here $\delta_{X}=i_{X} \delta+\delta i_{X}$ denotes the Lie derivative along the evolutionary vector field $X$ on the jet space of fields and antifields, while $L_{\xi}=d i_{\xi}+i_{\xi} d$ is the usual Lie derivative on horizontal forms. Using Proposition 5.1, one can easily see that $X$ is a symmetry of the gauge system, i.e., $[Q, X]=0$. Furthermore, this symmetry is Hamiltonian relative to (5.4):

$$
i_{X} \omega \simeq \delta \Xi
$$

where

$$
\Xi=-A^{*} \wedge L_{\xi} A-C^{*} \wedge L_{\xi} C, \quad \operatorname{gh}(\Xi)=-1 .
$$

The Hamiltonian $\Xi$ generates the symmetry transformations (5.7) through the BV bracket

$$
\delta_{X} \Phi=-\{\Xi, \Phi\}, \quad \Phi=\left(A, C, A^{*}, C^{*}\right) .
$$

By Proposition 3.7, $\Xi$ is an observable. We have

$$
\delta_{Q} \Xi=d \Theta, \quad \Theta \approx \frac{1}{2}\left(F \wedge i_{\xi} \tilde{F}-\tilde{F} \wedge i_{\xi} F\right), \quad \operatorname{gh}(\Theta)=0 .
$$

Thus, to each Killing vector we can associate a conserved current $\Theta$. Using the Hodge dualization, we can write

$$
* \Theta=\xi^{\mu} T_{\mu \nu} d x^{\nu},
$$

where $\left\{x^{\nu}\right\}$ are local coordinates on $M$ and $T_{\mu \nu}=T_{\nu \mu}$ is nothing but the energy-momentum tensor of the electromagnetic field. Since $\delta_{Q} \Theta=0$, the observable $\Xi$ gives no lower-degree conservation laws. In other words, the length of the space-time symmetry $X$ is equal to 1 . 
The free electromagnetic field admits also a symmetry of length 2 . This is generated by the evolutionary vector field $Y$ defined by the relations

$$
\delta_{Y} C^{*}=0, \quad \delta_{Y} A^{*}=0, \quad \delta_{Y} A=0, \quad \delta_{Y} C=1 .
$$

One can easily check that $Y$ is a nontrivial Hamiltonian symmetry of the master Lagrangian (5.6), i.e.,

$$
[Q, Y]=0, \quad i_{Y} \omega=\delta C^{*}, \quad \mathcal{L}_{Y} L \simeq 0 .
$$

The symmetry owes its origin to the global reducibility [5, 29] of the gauge transformations $\delta_{\varepsilon} A=d \varepsilon$, meaning that we can shift the gauge parameter $\varepsilon$ by an arbitrary constant for no change of $\delta_{\varepsilon} A$. By Proposition 3.7, $C^{*}$ is an observable giving rise to the sequence of conservation laws

$$
\delta_{Q} C^{*}=d A^{*}, \quad \delta_{Q} A^{*}=d \tilde{F}, \quad \delta_{Q} \tilde{F}=0 .
$$

As is seen, the forms $A^{*}$ and $\tilde{F}$ define the conserved currents of degrees 3 and 2 , so that the length of the symmetry $Y$ is 2 . The latter conserved current has ghost number zero and expresses Gauss' law:

$$
q=\int_{S} \tilde{F}
$$

In words, it states that the net electric flux through any closed, space-like surface $S$ is equal to the net electric charge $q$ within that closed surface.

\subsection{The abelian Chern-Simons theory}

Consider now the Chern-Simons theory for a trivial $U(1)$-bundle over a 3-dimensional manifold $M$. The theory is known to be purely gauge, possessing no local degrees of freedom. The spectrum of the BV fields and antifields is given by the gauge potential $A \in \Lambda^{1}(M)$, ghost field $C \in \Lambda^{0}(M)$ and their conjugate antifields $A^{*} \in \Lambda^{2}(M)$ and $C^{*} \in \Lambda^{3}(M)$. These are prescribed the following ghost numbers:

$$
\operatorname{gh}\left(C^{*}\right)=-2, \quad \operatorname{gh}\left(A^{*}\right)=-1, \quad \operatorname{gh}(A)=0, \quad \operatorname{gh}(C)=1 .
$$

The classical BRST differential $Q$ acts in the space of fields and antifields according to the relations

$$
\delta_{Q} C^{*}=d A^{*}, \quad \delta_{Q} A^{*}=d A, \quad \delta_{Q} A=d C, \quad \delta_{Q} C=0 .
$$

This action is Hamiltonian with respect to the canonical BV symplectic structure

$$
\omega=\delta A \wedge \delta A^{*}+\delta C \wedge \delta C^{*}, \quad \operatorname{gh}(\omega)=-1
$$

and the Hamiltonian for $Q$ is given by the ghost-extended Chern-Simons' Lagrangian

$$
L=\frac{1}{2} A \wedge d A+d C \wedge A^{*}, \quad i_{Q} \omega \simeq \delta L .
$$

As usual the BRST differential of $L$ gives rise to the conserved BRST current $L_{1}^{\prime}=2 L_{1}$, where

$$
L_{1}=\frac{3}{2} C \wedge d A, \quad d L_{1}=\delta_{Q} L
$$


This current is necessarily trivial. Starting from the BV symplectic structure (5.9), one can define the full sequence of descendent presymplectic structures of increasing ghost number:

$$
\begin{array}{lll}
\delta_{Q} \omega=d \omega_{1}, & \omega_{1}=\frac{1}{2} \delta A \wedge \delta A+\delta C \wedge \delta A^{*}, & \operatorname{gh}\left(\omega_{1}\right)=0, \\
\delta_{Q} \omega_{1}=d \omega_{2}, & \omega_{2}=\delta C \wedge \delta A, & \operatorname{gh}\left(\omega_{2}\right)=1, \\
\delta_{Q} \omega_{2}=d \omega_{3}, & \omega_{3}=\frac{1}{2} \delta C \wedge \delta C, & \operatorname{gh}\left(\omega_{3}\right)=2 .
\end{array}
$$

In particular, the BRST current $L_{1}^{\prime}$ obeys the master equation

$$
\left\{L_{1}^{\prime}, L_{1}^{\prime}\right\}_{1} \simeq 0
$$

relative to the Lie bracket associated with $\omega_{1}$.

Notice that the gauge symmetry transformations for the Chern-Simons field, being identical in form to those of the electromagnetic field, are globally reducible and this leads to the odd symmetry $Y$. Explicitly,

$$
\delta_{Y} C^{*}=0, \quad \delta_{Y} A^{*}=0, \quad \delta_{Y} A=0, \quad \delta_{Y} C=1 .
$$

The symmetry is obviously Hamiltonian,

$$
i_{Y} \omega=\delta C^{*},
$$

and the Hamiltonian $C^{*}$ gives rise to the conserved currents $A^{*}, A$, and $C$ as is seen from (5.8). Computing the various Lie brackets of the currents, we find

$$
\left\{A^{*}, A^{*}\right\}_{1}=0, \quad\{A, A\}_{2}=0, \quad\{C, C\}_{3}=-1 .
$$

Here we face with the phenomenon of central extension mentioned at the end of Section 4. Namely, the abelian super-Lie algebra $[Y, Y]=0$ of symmetry gets central extension when evaluated at the level of conserved currents.

The integral of the conserved current $A$ over a loop $\gamma \subset M$ gives the conserved "charge"

$$
h=\int_{\gamma} A
$$

which is nothing but the holonomy of the flat abelian connection $A$. One can think of these holonomy invariants as global degrees of freedom in the Chern-Simons theory.

\subsection{Linearized gravity}

Our last example is the free massless field of spin 2. This theory can be obtained by linearizing Einstein's equations about the flat background. In the vierbein formalism, the background geometry is described by the vierbein $\left\{h^{a}\right\}$, which is assumed to be given by a set of four linearly independent, closed 1-forms on the Minkowski space. The small fluctuations of geometry around the flat background are described by the collection of ten 1-form fields $e^{a}$ and $\omega^{a b}=-\omega^{b a}$. These are identified, respectively, with the perturbations of the flat vierbein and spin connection. In accordance with the general prescriptions of the BV formalism, this set of fields is extended by the ghost fields $c^{a}$ and $c^{a b}=-c^{b a}$, associated with the general coordinate and local Lorentz invariance, as well as the antifields $e_{a}^{*}, \omega_{a b}^{*}, c_{a}^{*}$, and $c_{a b}^{*}$. The form degrees and the ghost numbers of the introduced fields are collected in the following table:

\begin{tabular}{|c|c|c|c|c|c|c|c|c|}
\hline & $c_{a}^{*}$ & $c_{a b}^{*}$ & $e_{a}^{*}$ & $\omega_{a b}^{*}$ & $e^{a}$ & $\omega^{a b}$ & $c^{a}$ & $c^{a b}$ \\
\hline $\operatorname{deg}$ & 4 & 4 & 3 & 3 & 1 & 1 & 0 & 0 \\
\hline gh & -2 & -2 & -1 & -1 & 0 & 0 & 1 & 1 \\
\hline
\end{tabular}


The canonical BV symplectic structure assumes the form

$$
\omega=\delta e^{a} \wedge \delta e_{a}^{*}+\delta \omega^{a b} \wedge \delta \omega_{a b}^{*}+\delta c^{a} \wedge \delta c_{a}^{*}+\delta c^{a b} \wedge \delta c_{a b}^{*} .
$$

In order to define the classical BRST differential $Q$ it is convenient to introduce the following collections of background 1- and 2-forms:

$$
h_{a b c}=\varepsilon_{a b c d} h^{d}, \quad H_{a b}=h^{c} \wedge h_{a b c},
$$

with $\varepsilon_{a b c d}$ being the Levi-Civita symbol. Then the action of $Q$ is given by the relations

$$
\begin{array}{ll}
\delta_{Q} c_{a}^{*}=d e_{a}^{*}, & \delta_{Q} c_{a b}^{*}=d \omega_{a b}^{*}-\frac{1}{2}\left(e_{a}^{*} \wedge h_{b}-e_{b}^{*} \wedge h_{a}\right), \\
\delta_{Q} e_{a}^{*}=d \omega^{b c} \wedge h_{a b c}, & \delta_{Q} \omega_{a b}^{*}=d e^{c} \wedge h_{c a b}+\frac{1}{2}\left(\omega_{b}^{c} \wedge H_{a c}-\omega_{a}^{c} \wedge H_{b c}\right), \\
\delta_{Q} e^{a}=d c^{a}+c^{a b} h_{b}, & \delta_{Q} \omega^{a b}=d c^{a b}, \\
\delta_{Q} c^{a}=0, & \delta_{Q} c^{a b}=0 .
\end{array}
$$

This action is Hamiltonian relative to (5.10) and is generated by the BV master Lagrangian

$$
L=e^{a} \wedge d \omega^{b c} \wedge h_{a b c}+\frac{1}{2} \omega^{a}{ }_{c} \wedge \omega^{c b} \wedge H_{a b}+e_{a}^{*} \wedge\left(d c^{a}+c^{a b} h_{b}\right)+\omega_{a b}^{*} \wedge d c^{a b} .
$$

Hereafter all indices are raised and lowered by means of the Minkowski metric. The BV symplectic structure (5.10) gives rise to the following sequence of presymplectic forms of decreasing horizontal degree and increasing ghost number:

$$
\begin{aligned}
& \delta_{Q} \omega=d \omega_{1}, \quad \omega_{1}=\delta e^{a} \wedge \delta \omega^{b c} \wedge h_{a b c}+\delta c^{a} \wedge \delta e_{a}^{*}+\delta c^{a b} \wedge \delta \omega_{a b}^{*}, \quad \operatorname{gh}\left(\omega_{1}\right)=0, \\
& \delta_{Q} \omega=d \omega_{2}, \quad \omega_{2}=\left(\delta c^{a} \wedge \delta \omega^{b c}+\delta c^{a b} \wedge \delta e^{c}\right) \wedge h_{a b c}, \quad \operatorname{gh}\left(\omega_{2}\right)=1, \\
& \delta_{Q} \omega=d \omega_{3}, \quad \omega_{3}=\delta c^{a} \wedge \delta c^{b c} \wedge h_{a b c}, \quad \operatorname{gh}\left(\omega_{3}\right)=2,
\end{aligned}
$$

and $\delta_{Q} \omega_{3}=0$. Thus, the length of the linearized gravity is 3 .

As with the Maxwell electrodynamics, the isometries of the Minkowski space give rise to the conserved energy-momentum tensor of spin-2 field. This conservation law, however, does not survive in the full nonlinear theory. In general relativity, the canonical energy-momentum tensor is known to vanish on shell. Much more interesting are the lower-degree conservation laws that are present in the theory. These can be constructed as follows.

Let $\xi^{a}$ and $\xi^{a b}=-\xi^{b a}$ be some functions on the Minkowski space. Define the odd evolutionary vector field $Y$ by the relations

$$
\delta_{Y} c^{a}=\xi^{a}, \quad \delta_{Y} c^{a b}=\xi^{a b}, \quad \delta_{Y}(\text { the other fields })=0 .
$$

As above, by $\delta_{Y}$ we denoted the Lie derivative along $Y$. Using Proposition 5.1, one can see that the vector field $Y$ is a symmetry iff the following equations are satisfied:

$$
d \xi^{a}=\xi^{a b} h_{b}, \quad d \xi^{a b}=0 .
$$

The general solution to these equations is obvious. If we choose $h^{a}=d x^{a}$, where $x^{a}$ are the Cartesian coordinates on $\mathbb{R}^{1,3}$, then

$$
\xi^{a}(x)=\zeta^{a}+\zeta^{a b} x_{b}, \quad \xi^{a b}(x)=\zeta^{a b}
$$

for arbitrary constant parameters $\zeta^{a}$ and $\zeta^{a b}=-\zeta^{b a}$. The $\xi^{\prime}$ 's are naturally identified with the ten Killing vectors of the Minkowski metric. The symmetry $Y$ is clearly Hamiltonian:

$$
i_{Y} \omega=\delta H, \quad H=\xi^{a} c_{a}^{*}+\xi^{a b} c_{a b}^{*} .
$$


The Hamiltonian $H$, being a physical observable, generates the following sequence of conserved currents:

$$
\begin{aligned}
& \delta_{Q} H=d J_{1}, \quad J_{1}=\xi^{a} e_{a}^{*}+\xi^{a b} \omega_{a b}^{*}, \quad \operatorname{deg} J_{1}=3, \quad \operatorname{gh} J_{1}=-1, \\
& \delta_{Q} J_{1}=d J_{2}, \quad J_{2}=\left(\xi^{a} \omega^{b c}+\xi^{a b} e^{c}\right) \wedge h_{a b c}, \quad \operatorname{deg} J_{2}=2, \quad \operatorname{gh} J_{2}=0, \\
& \delta_{Q} J_{2}=d J_{3}, \quad J_{3}=\left(\xi^{a} c^{b c}+\xi^{a b} c^{c}\right) \wedge h_{a b c}, \quad \operatorname{deg} J_{3}=1, \quad \operatorname{gh} J_{3}=1,
\end{aligned}
$$

and $\delta_{Q} J_{3}=0$. The integral of the 10-parameter family of conserved currents $J_{2}$ over a closed, space-like surface $S \subset \mathbb{R}^{1,3}$ gives the net energy-momentum $\mathcal{P}$ and the angular momentum $\mathcal{M}$ of the spin-2 field produced by the sources inside $S$ :

$$
\mathcal{P}_{a}=\int_{S} \omega^{b c} \wedge h_{a b c}, \quad \mathcal{M}_{a b}=\int_{S} e^{c} \wedge h_{a b c}+\frac{1}{2}\left(x_{b} \omega^{d c} \wedge h_{a d c}-x_{a} \omega^{d c} \wedge h_{b d c}\right) .
$$

Although these conserved currents do not extend into the full nonlinear theory of gravity, they can be used for the derivation of asymptotic conservation laws (e.g., ADM energy) in general relativity via a surface integral at infinity [3, 4, 29].

Evaluating now the descendent brackets of the conserved currents above, one can easily find

$$
\left\{J_{1}^{\xi}, J_{1}^{\xi^{\prime}}\right\}_{1}=0, \quad\left\{J_{2}^{\xi}, J_{2}^{\xi^{\prime}}\right\}_{2}=0, \quad\left\{J_{3}^{\xi}, J_{3}^{\xi^{\prime}}\right\}_{3}=-\left(\xi^{a} \xi^{\prime b c}+\xi^{\prime a} \xi^{b c}\right) h_{a b c}
$$

As with the Chern-Simons theory, the Lie brackets of the zero-degree currents get the central extension.

\section{A Jet bundles and the variational bicomplex}

In this appendix, we briefly recall some basic elements from the theory of jet bundles and variational bicomplex, which are relevant for our discussion. A more systematic exposition of these concepts can be found in [2, 11, 24, 26].

The starting point of any field theory is a locally trivial fiber bundle $\pi: E \rightarrow M$ whose base is identified with the space-time manifold and which sections are called classical fields. For the sake of simplicity, we restrict ourselves to fields with values in vector bundles, although the subsequent discussion could be straightforwardly extended to general smooth bundles. On the other hand, to accommodate bosonic and fermionic fields, we allow the fibers of $E$ to be superspaces with a given number of even and odd dimensions; in so doing, the base $M$ remains a pure even manifold. The Grassmann parity of a homogeneous object $A$ is denoted by $\epsilon(A) \in\{0,1\}$.

Associated with a vector bundle $\pi: E \rightarrow M$ is the vector bundle $\pi_{k}: J^{k} E \rightarrow M$ of $k$-jets of sections of $E$. By definition, the $k$-jet $j_{x}^{k} \phi$ at $x \in M$ is just the equivalence class of the section $\phi \in \Gamma(E)$, where two sections are considered to be equivalent if they have the same Taylor development of order $k$ at $x \in M$ in some (and hence any) adapted coordinate chart. It follows from the definition that each section $\phi$ of $E$ induces the section $j^{k} \phi$ of $J^{k} E$ by the rule $\left(j^{k} \phi\right)(x)=j_{x}^{k} \phi$. The latter is called the $k$-jet prolongation of $\phi$.

If $\left.E\right|_{U} \simeq \mathbb{R}^{m} \times U$ is an adapted coordinate chart with local coordinates $\left(x^{i}, \phi^{a}\right)$, then $\left(x^{i}, \phi^{a}, \phi_{i}^{a}, \ldots, \phi_{i_{1} \cdots i_{k}}^{a}\right)$ are local coordinates in $J^{k} E$ and the induced section $j^{k} \phi$ is given in these coordinates by

$$
x \mapsto\left(x, \phi^{a}(x), \partial_{i} \phi^{a}(x), \ldots, \partial_{i_{1}} \cdots \partial_{i_{k}} \phi^{a}(x)\right) .
$$

We use the multi-index notation and the summation convention through the paper. A multiindex $I=i_{1} i_{2} \cdots i_{n}$ represents the corresponding set of symmetric covariant indices. The order 
of the multi-index is given by $|I|=k$. By definition we set $I j=j I=i_{1} i_{2} \cdots i_{k} j$. With the multi-index notation we can write the partial derivatives of fields as $\partial_{i_{1}} \cdots \partial_{i_{k}} \phi^{a}=\partial_{I} \phi^{a}$ and the set of local coordinates on $\left.J^{k} E\right|_{U}$ as $\left(x^{i}, \phi_{I}^{a}\right),|i| \leq k$.

Jet bundles come with natural projection $J^{k} E \rightarrow J^{k-1} E$ defined by forgetting all the derivatives of order $k$. One can easily see that this projection gives $J^{k} E$ the structure of an affine bundle over the base $J^{k-1} E$. Thus, we have the infinite sequence of surjective submersions

$$
\cdots \longrightarrow J^{3} E \longrightarrow J^{2} E \longrightarrow J^{1} E \longrightarrow J^{0} E \simeq E
$$

The infinite order jet bundle $J^{\infty} E$ is now defined as the inverse limit over the jet order $k$ :

$$
J^{\infty} E=\lim _{\longleftarrow} J^{k} E .
$$

Let $\Lambda^{*}\left(J^{k} E\right)$ denote the space of differential forms on $J^{k} E$. The sequence of projections (A.1) gives rise to the chain of pullback maps

$$
\cdots \longleftarrow \Lambda^{*}\left(J^{3} E\right) \longleftarrow \Lambda^{*}\left(J^{2} E\right) \longleftarrow \Lambda^{*}\left(J^{1} E\right) \longleftarrow \Lambda^{*}\left(J^{0} E\right) .
$$

This allows one to define the space of differential forms on $\Lambda\left(J^{\infty} E\right)$ as the direct limit

$$
\Lambda^{*}\left(J^{\infty} E\right)=\lim _{\longrightarrow} \Lambda^{*}\left(J^{k} E\right)
$$

According to this definition each differential form on $J^{\infty} E$ is the pullback of a smooth form on some finite jet bundle $J^{k} E$. As usual, the smooth functions on $J^{\infty} E$ are identified with the 0 -forms. For notational simplicity, we will not distinguish between a form on $J^{\infty} E$ and its representatives in finite-dimensional jet bundles. The exterior differential on $\Lambda^{*}\left(J^{\infty} E\right)$ will be denoted by $D$.

The de Rham complex $\left(\Lambda^{*}\left(J^{\infty} E\right), D\right)$ of differential forms on $J^{\infty} E$ possesses the differential ideal $\mathcal{C}\left(J^{\infty} E\right)$ of contact forms. By definition, $\alpha \in \mathcal{C}\left(J^{\infty} E\right)$ iff $\left(j^{\infty} \phi\right)^{*} \alpha=0$ for all sections $\phi \in \Gamma(E)$. The ideal $\mathcal{C}\left(J^{\infty} E\right)$ is known to be generated by the contact 1-forms, which in local coordinates take the form $\delta \phi_{I}^{a}:=D \phi_{I}^{a}-\phi_{I j}^{a} D x^{j}$. Using the contact forms, one can split the exterior differential $D$ into the sum of horizontal and vertical differentials, namely, $D=d+\delta$ where

$$
d=d x^{j} \wedge\left(\frac{\partial}{\partial x^{j}}+\phi_{I j}^{a} \frac{\partial}{\partial \phi_{I}^{a}}\right), \quad \delta=\delta \phi_{I}^{a} \wedge \frac{\partial_{l}}{\partial \phi_{I}^{a}} .
$$

It is easy to see that

$$
d^{2}=0, \quad \delta^{2}=0, \quad d \delta+\delta d=0 .
$$

Any $p$-form of $\Lambda^{p}\left(J^{\infty} E\right)$ can now be written as a finite sum of homogeneous forms

$$
f d x^{i_{1}} \wedge \cdots \wedge d x^{i_{r}} \wedge \delta \phi_{I_{1}}^{a_{1}} \wedge \cdots \wedge \delta \phi_{I_{s}}^{a_{s}}
$$

of horizontal degree $r$ and vertical degree $s$, with $r+s=p$ and $f$ being a smooth function on $J^{\infty} E$. The variational bicomplex is the double complex $\left(\Lambda^{*, *}\left(J^{\infty} E\right), \delta, d\right)$ of differential 
forms on $J^{\infty} E$ :

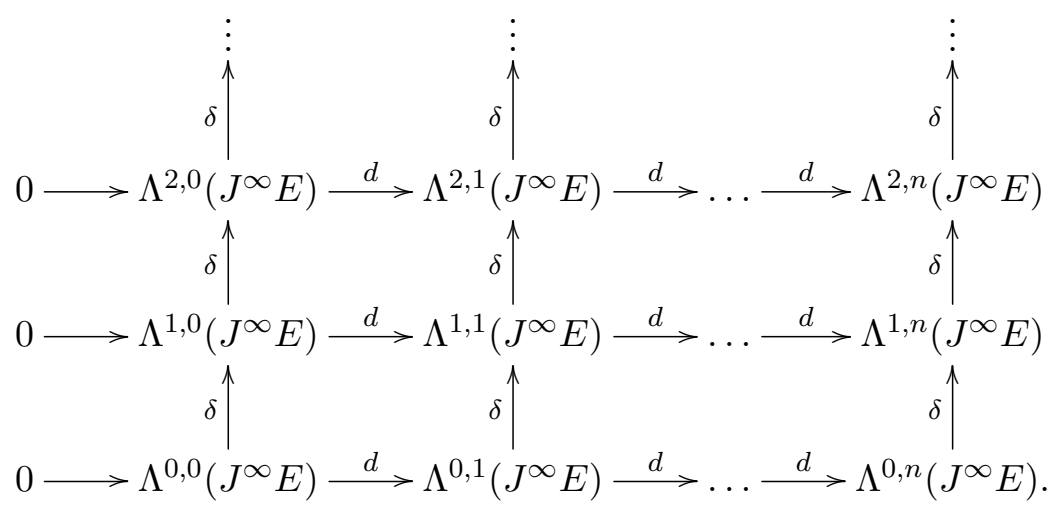

The most important result concerning the variational bicomplex for the vector bundle $\pi: E \rightarrow M$ is that all the columns and interior rows of the diagram above are exact.

It is possible to augment the variational bicomplex from below by the de Rham complex of the base manifold:

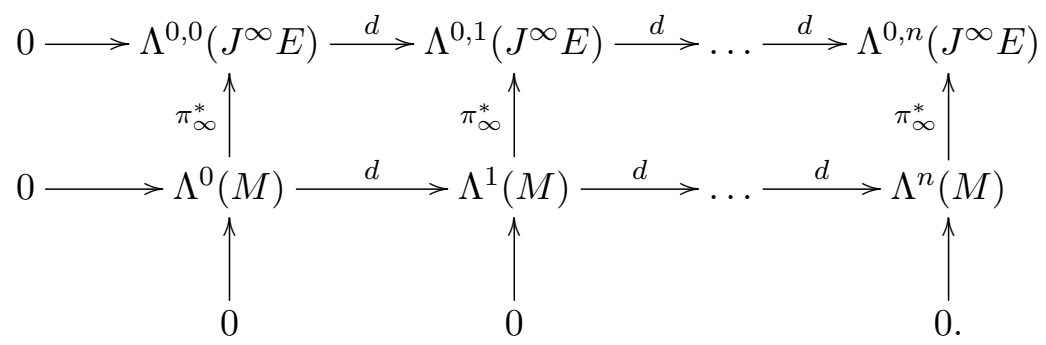

The resulting bicomplex remains exact in columns.

As with any bicomplex, one can consider the relative cohomology of " $\delta$ modulo $d$ ". It is described by the groups $H^{p, q}\left(J^{\infty} E ; \delta / d\right)$ which are essentially the cohomology groups of the quotient complex $\widetilde{\Lambda}^{p, q}\left(J^{\infty} E\right)=\Lambda^{p, q}\left(J^{\infty} E\right) / d \Lambda^{p, q-1}\left(J^{\infty} E\right)$ with differential induced by $\delta$. In the main text, we often use the following statement about the relative $\delta$-cohomology.

Proposition A.1 ([11, Section 19.3.9]).

$$
H^{p, q}\left(J^{\infty} E ; \delta / d\right)=0 \quad \text { for } \quad p>0 \quad \text { and } \quad H^{0, q}\left(J^{\infty} E ; \delta / d\right) \simeq \Lambda^{q}(M) / d \Lambda^{q-1}(M) .
$$

The quotient $\delta$-complex $\widetilde{\Lambda}^{p, n}\left(J^{\infty} E\right)=\Lambda^{p, n}\left(J^{\infty} E\right) / d \Lambda^{p, n-1}\left(J^{\infty} E\right)$ provides a natural augmentation of the variational bicomplex from the right:

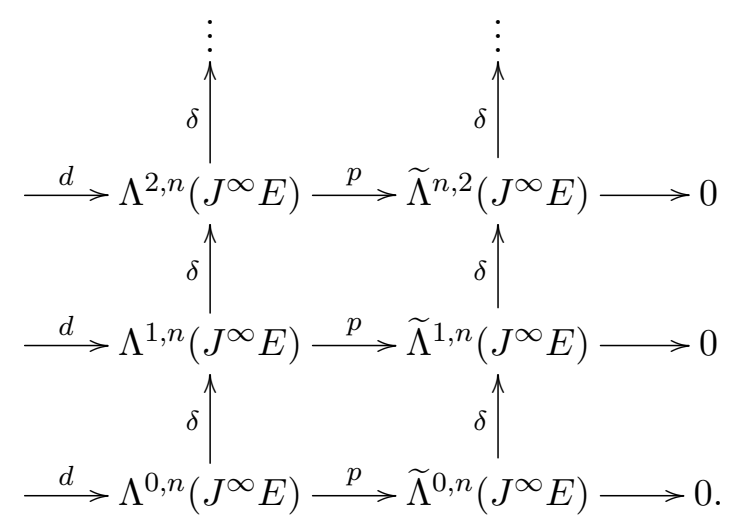


$p$ being the canonical projection onto the quotient space. Proposition A.1 ensures that the appended column is exact. The space $\widetilde{\Lambda}^{0, n}\left(J^{\infty} E\right)$ is usually identified with the space of local functionals of fields. The correspondence between the two spaces is established by the assignment

$$
\widetilde{\Lambda}^{0, n}\left(J^{\infty} E\right) \ni[a] \mapsto A[\phi]=\int_{M}\left(j^{\infty} \phi\right)^{*}(a),
$$

with $\phi$ being a compactly supported section of $E$.

The space $\Lambda^{1, n}\left(J^{\infty} E\right)$ has a distinguished subspace spanned by the source forms. These are given by finite sums of the forms

$$
\alpha \wedge \delta \phi^{a}
$$

where $\alpha \in \Lambda^{0, n}\left(J^{\infty} E\right)$. Using the exactness of the variational bicomplex one can prove the following

Proposition A.2 ([11]). For any $(1, n)$-form $\alpha$ there exists a unique source form $\beta$ and $a(1, n-$ 1)-form $\gamma$ such that

$$
\alpha=\beta+d \gamma .
$$

The form $\gamma$ is uniquely determined up to a d-exact form. In particular, a nonzero source form can never be d-exact.

Given $\lambda \in \Lambda^{0, n}\left(J^{*} E\right)$, we can apply the proposition above to $\delta \lambda$. We get

$$
\delta \lambda=\delta \phi^{a} \wedge \frac{\delta \lambda}{\delta \phi^{a}}+d \gamma
$$

The coefficients $\delta \lambda / \delta \phi^{a}$ defining the source form are called the Euler-Lagrange derivative of the form $\lambda$. Explicitly,

$$
\frac{\delta \lambda}{\delta \phi^{a}}=(-\partial)_{I} \frac{\partial \lambda}{\partial \phi_{I}^{a}}
$$

where

$$
(-\partial)_{I}=(-1)^{|I|} \partial_{I}, \quad \partial_{I}=\partial_{i_{1}} \cdots \partial_{i_{k}}, \quad \partial_{i}=\frac{\partial}{\partial x^{i}}+\phi_{I i}^{a} \frac{\partial_{l}}{\partial \phi_{I}^{a}} .
$$

Dual to the space of 1 -forms on $J^{\infty} E$ is the space of vector fields $\mathfrak{X}\left(J^{\infty} E\right)$. In terms of local coordinates, the elements of $\mathfrak{X}\left(J^{\infty} E\right)$ are given by the formal series

$$
X=X^{i} \frac{\partial}{\partial x^{i}}+X_{I}^{a} \frac{\partial_{l}}{\partial \phi_{I}^{a}}
$$

where $X^{i}$ and $X_{I}^{a}$ are smooth functions on $J^{\infty} E$. A vector field $X$ is called vertical if $X^{i}=0$.

The operation $i_{X}$ of contraction of the vector field (A.3) with a differential form is defined as usual: $i_{X}$ is a differentiation of the exterior algebra $\Lambda^{*}\left(J^{\infty} E\right)$ of form degree -1 and the Grassmann parity $\widetilde{X}+1$ which action on the basis 1 -forms is given by

$$
i_{X} \delta \phi_{I}^{a}=X_{I}^{a}, \quad i_{X} d x^{j}=X^{j} .
$$

The operator of the Lie derivative along the vector field $X$ is defined by the magic Cartan's formula

$$
L_{X}=i_{X} D+(-1)^{\epsilon(X)} D i_{X} .
$$


A vertical vector field $X$ is called evolutionary if

$$
i_{X} d+(-1)^{\epsilon(X)} d i_{X}=0 .
$$

It follows from the definition that the vector field (A.3) is evolutionary iff $X^{i}=0$ and $X_{I}^{a}=$ $\partial_{I}\left(X^{a}\right)$, where $\partial_{I}$ is defined by (A.2). Hence, any vertical field of the form $X_{0}=X^{a} \partial / \partial \phi^{a}$ admits a unique prolongation to an evolutionary vector field. We call $X_{0}$ the source vector field for the evolutionary vector field $X$. (Our nomenclature is not standard; most of the authors prefer to call the vector field $X_{0}$ evolutionary, rather than its prolongation $X$.) Note that the Lie derivative along the evolutionary vector field $X$ can be written as $L_{X}=i_{X} \delta+(-1)^{\epsilon(X)} \delta i_{X}$. It is clear that the evolutionary vector fields form a closed Lie algebra.

\section{Acknowledgements}

The work was partially supported by the RFBR grant No. 16-02-00284 A.

\section{References}

[1] Alkalaev K.B., Grigoriev M., Frame-like Lagrangians and presymplectic AKSZ-type sigma models, Internat. J. Modern Phys. A 29 (2014), 1450103, 33 pages, arXiv:1312.5296.

[2] Anderson I.M., Introduction to the variational bicomplex, in Mathematical Aspects of Classical Field Theory (Seattle, WA, 1991), Contemp. Math., Vol. 132, Amer. Math. Soc., Providence, RI, 1992, 51-73.

[3] Anderson I.M., Torre C.G., Asymptotic conservation laws in classical field theory, Phys. Rev. Lett. 77 (1996), 4109-4113, hep-th/9608008.

[4] Barnich G., Brandt F., Covariant theory of asymptotic symmetries, conservation laws and central charges, Nuclear Phys. B 633 (2002), 3-82, hep-th/0111246.

[5] Barnich G., Brandt F., Henneaux M., Local BRST cohomology in gauge theories, Phys. Rep. 338 (2000), 439-569, hep-th/0002245.

[6] Barnich G., Henneaux M., Isomorphisms between the Batalin-Vilkovisky antibracket and the Poisson bracket, J. Math. Phys. 37 (1996), 5273-5296, hep-th/9601124.

[7] Bridges T.J., Hydon P.E., Lawson J.K., Multisymplectic structures and the variational bicomplex, Math. Proc. Cambridge Philos. Soc. 148 (2010), 159-178.

[8] Bryant R.L., Griffiths P.A., Characteristic cohomology of differential systems. I. General theory, J. Amer. Math. Soc. 8 (1995), 507-596.

[9] Cattaneo A.S., Schätz F., Introduction to supergeometry, Rev. Math. Phys. 23 (2011), 669-690, arXiv:1011.3401.

[10] Crnković Č., Witten E., Covariant description of canonical formalism in geometrical theories, in Three Hundred Years of Gravitation, Cambridge University Press, Cambridge, 1987, 676-684.

[11] Dickey L.A., Soliton equations and Hamiltonian systems, Advanced Series in Mathematical Physics, Vol. 12, World Scientific Publishing Co., Inc., River Edge, NJ, 1991.

[12] Grigoriev M., Presymplectic structures and intrinsic Lagrangians, arXiv:1606.07532.

[13] Grigoriev M.A., Semikhatov A.M., Tipunin I.Yu., Becchi-Rouet-Stora-Tyutin formalism and zero locus reduction, J. Math. Phys. 42 (2001), 3315-3333, hep-th/0001081.

[14] Henneaux M., Knaepen B., Schomblond C., Characteristic cohomology of p-form gauge theories, Comm. Math. Phys. 186 (1997), 137-165, hep-th/9606181.

[15] Henneaux M., Teitelboim C., Quantization of gauge systems, Princeton University Press, Princeton, NJ, 1992.

[16] Kaparulin D.S., Lyakhovich S.L., Sharapov A.A., Rigid symmetries and conservation laws in non-Lagrangian field theory, J. Math. Phys. 51 (2010), 082902, 22 pages, arXiv:1001.0091.

[17] Kaparulin D.S., Lyakhovich S.L., Sharapov A.A., Local BRST cohomology in (non-)Lagrangian field theory, J. High Energy Phys. 2011 (2011), no. 9, 006, 34 pages, arXiv:1106.4252. 
[18] Kazinski P.O., Lyakhovich S.L., Sharapov A.A., Lagrange structure and quantization, J. High Energy Phys. 2005 (2005), no. 7, 076, 42 pages, hep-th/0506093.

[19] Khavkine I., Presymplectic current and the inverse problem of the calculus of variations, J. Math. Phys. 54 (2013), 111502, 11 pages, arXiv:1210.0802.

[20] Khavkine I., Covariant phase space, constraints, gauge and the Peierls formula, Internat. J. Modern Phys. A 29 (2014), 1430009, 74 pages, arXiv:1402.1282.

[21] Kosmann-Schwarzbach Y., The Noether theorems. Invariance and conservation laws in the twentieth century, Sources and Studies in the History of Mathematics and Physical Sciences, Springer, New York, 2011.

[22] Lyakhovich S.L., Sharapov A.A., BRST theory without Hamiltonian and Lagrangian, J. High Energy Phys. 2005 (2005), no. 3, 011, 22 pages, hep-th/0411247.

[23] Mehta R.A., Supergroupoids, double structures, and equivariant cohomology, Ph.D. Thesis, University of California, Berkeley, 2006, math.DG/0605356.

[24] Olver P.J., Applications of Lie groups to differential equations, Graduate Texts in Mathematics, Vol. 107, Springer-Verlag, New York, 1986.

[25] Roytenberg D., On the structure of graded symplectic supermanifolds and Courant algebroids, in Quantization, Poisson Brackets and Beyond (Manchester, 2001), Contemp. Math., Vol. 315, Amer. Math. Soc., Providence, RI, 2002, 169-185, math.SG/0203110.

[26] Saunders D.J., The geometry of jet bundles, London Mathematical Society Lecture Note Series, Vol. 142, Cambridge University Press, Cambridge, 1989.

[27] Sharapov A.A., Variational tricomplex of a local gauge system, Lagrange structure and weak Poisson bracket, Internat. J. Modern Phys. A 30 (2015), 1550152, 32 pages, arXiv:1506.04652.

[28] Sharapov A.A., On presymplectic structures for massless higher-spin fields, Eur. Phys. J. C Part. Fields 76 (2016), 305, 16 pages, arXiv:1602.06393.

[29] Torre C.G., Local cohomology in field theory (with applications to the Einstein equations), Lectures given at 2nd Mexican School on Gravitation and Mathematical Physics (December 1-7, 1996, Tlaxcala, Mexico), hep-th/9706092.

[30] Tsujishita T., Homological method of computing invariants of systems of differential equations, Differential Geom. Appl. 1 (1991), 3-34.

[31] Verbovetsky A., Notes on the horizontal cohomology, in Secondary Calculus and Cohomological Physics (Moscow, 1997), Contemp. Math., Vol. 219, Amer. Math. Soc., Providence, RI, 1998, 211-231, math.DG/9803115.

[32] Vinogradov A.M., The $\mathcal{C}$-spectral sequence, Lagrangian formalism, and conservation laws. I. The linear theory, J. Math. Anal. Appl. 100 (1984), 1-40.

[33] Vinogradov A.M., The $\mathcal{C}$-spectral sequence, Lagrangian formalism, and conservation laws. II. The nonlinear theory, J. Math. Anal. Appl. 100 (1984), 41-129.

[34] Voronov T., Graded manifolds and Drinfeld doubles for Lie bialgebroids, in Quantization, Poisson Brackets and Beyond (Manchester, 2001), Contemp. Math., Vol. 315, Amer. Math. Soc., Providence, RI, 2002, 131168, math.DG/0105237.

[35] Zuckerman G.J., Action principles and global geometry, in Mathematical Aspects of String Theory (San Diego, Calif., 1986), Adv. Ser. Math. Phys., Vol. 1, World Sci. Publishing, Singapore, 1987, 259-284. 